\title{
A comprehensive approach to the bioavailability and cardiometabolic effects of the bioactive compounds present in espresso coffee and confectionery-derived coffee
}

\author{
Pedro Mena $^{1}$, Michele Tassotti ${ }^{1}$, Alice Rosi ${ }^{1}$, Daniela Martini ${ }^{1}$, Laura Righetti ${ }^{1}$, \\ Monica Antonini ${ }^{2}$, Margherita Dall'Asta ${ }^{1}$, Letizia Bresciani ${ }^{1}$, Federica Fantuzzi ${ }^{2}$ \\ Valentina Spigoni ${ }^{2}$, Angel Gil-Izquierdo ${ }^{3}$, Raúl Domínguez-Perles, ${ }^{3}$, Pedro Luis Tornel ${ }^{4}$, \\ Soledad Del Pozo-Luengo 4 , Donato Angelino', Cristian Del Bo,5, Chiara Dall'Asta1 ${ }^{1}$, \\ Patrizia Riso ${ }^{5}$, Alessandra Dei Cas ${ }^{2}$, Riccardo Bonadonna ${ }^{2}$, Furio Brighenti ${ }^{1}$ and \\ Daniele Del Rio ${ }^{1}$ \\ ${ }^{1}$ Department of Food \& Drugs, University of Parma, Parma, Italy, \\ ${ }^{2}$ Department of Medicine and Surgery, University of Parma, Parma, Italy, \\ ${ }^{3}$ Department of Food Science and Technology, CEBAS-CSIC, Murcia, Spain, \\ ${ }^{4}$ Clinical Analysis Service, Hospital Virgen de la Arrixaca, Murcia, Spain and \\ ${ }^{5}$ Department of Food, Environmental and Nutritional Sciences, Università degli Studi di Milano, Milan, Italy
}

\section{Abstract}

Coffee is an important source of bioactive compounds, including caffeine, trigonelline, and phenolic compounds. Several studies have highlighted the preventive effects of coffee consumption on major cardiometabolic diseases, but the impact of coffee dosage on markers of cardiometabolic risk is not well understood. Moreover, the pool of coffee-derived circulating metabolites in real-life settings is unknown. This study evaluated the bioavailability and effects on recognised cardiometabolic markers of coffee bioactives, considering different levels of consumption. An innovative experimental design, including both a chronic and an acute sub-study, and a comprehensive analytical approach were used.

A 3-arm, randomised, crossover trial was conducted in 21 healthy volunteers (age, $23 \pm 2 \mathrm{y}$; BMI, $\left.22.3 \pm 2.5 \mathrm{~kg} / \mathrm{m}^{2}\right)(\mathrm{Mena}$ et al., Trials 2017, 18, 527). Volunteers were assigned to consume 3 treatments for 4 weeks, including 1 cup of espresso coffee/day, 3 cups of espresso coffee/day, and 1 cup of espresso coffee plus 2 cocoa-based confectionary products containing-coffee twice per day. The last day of each treatment, blood and urine samples were collected at specific time points for 24 hours. Dietary intake, body weight, BMI, waist circumference, blood pressure, fasting glucose, insulin, LDL- and HDL-cholesterol, triglycerides, nitric oxide, inflammatory markers (IL-8, TNF $\alpha$, VEGF), trimethylamine- $N$-oxide (TMAO), DNA damage, DNA catabolites, and eicosanoids were assessed. The pool of coffee-derived circulating metabolites was also assessed in acute conditions. Untargeted metabolomics was performed.

Energy intake did not change among treatments after 4 weeks, while significant differences were observed in the intake of saturated fatty acids and carbohydrates. The effect of different coffee dosages on the set of cardiometabolic markers assessed was negligible. Plasma and urinary pharmacokinetic profiles were evaluated for 6 caffeine metabolites, 3 trigonelline derivatives, and up to 40 phenolic metabolites. Pharmacokinetics highlighted the different "waves" of circulating metabolites occurring upon repeated coffee consumption. Differences in several pharmacokinetic parameters were observed among treatments, which may support the long-term cardiometabolic benefits of certain patterns of coffee consumption. Multivariate analyses clearly differentiated treatments on the basis of the urinary metabolome.

This work provided a comprehensive picture of the impact of different coffee dosages on the pool of coffee-derived circulating metabolites, the urinary metabolome, and a wide number of cardiometabolic markers. Multivariate analyses focused on inter-individual differences are ongoing to better understand the effect of coffee on cardiometabolic health.

\section{Funding}

This study was funded by Soremartec Italia S.r.l. (Alba, Italy).

\section{Conflict of Interest}

There is no conflict of interest 